\section{$\underset{\substack{\text { hommes } \\ \text { \& migrations }}}{ }$}

\section{Hommes \& migrations}

Revue française de référence sur les dynamiques

migratoires

$1311 \mid 2015$

Femmes et migrations

\title{
Un jeu de ping-pong entre la France et le Liban
}

\section{Zeina Abirached et Hélène Bouillon}

\section{CpenEdition \\ Journals}

\section{Édition électronique}

URL : http://journals.openedition.org/hommesmigrations/3335

DOI : 10.4000/hommesmigrations.3335

ISSN : 2262-3353

\section{Éditeur}

Musée national de l'histoire de l'immigration

\section{Édition imprimée}

Date de publication : 1 juillet 2015

Pagination : 140-143

ISBN : 978-2-919040-32-2

ISSN : $1142-852 X$

\section{Référence électronique}

Zeina Abirached et Hélène Bouillon, «Un jeu de ping-pong entre la France et le Liban », Hommes \& migrations [En ligne], 1311 | 2015, mis en ligne le 09 février 2016, consulté le 15 septembre 2020. URL : http://journals.openedition.org/hommesmigrations/3335 


\section{COLLECTIONS}

\section{UN JEU DE PING-PONG ENTRE LA FRANCE ET LE LIBAN}

Entretien avec ZEINA ABIRACHED, auteure de bandes dessinées, par Hélène Bouillon, conservateure et responsable des collections historiques au Musée national de l'histoire de l'immigration.

\begin{abstract}
À l'occasion de l'exposition Albums, le Musée Ade l'histoire de l'immigration a acquis des planches de Zeina Abirached, née à Beyrouth en 1981 et qui vit en France depuis 2004. À travers ses bandes dessinées, elle interroge ses liens avec son pays d'origine, son bilinguisme, sa double culture en tant que femme enracinée et nomade à la fois.
\end{abstract}

\section{Hommes \& Migrations : Avez-vous depuis tou- jours voulu devenir dessinatrice de bande dessinée?}

Zeina Abirached: Absolument pas. C'est finalement arrivé assez tard. Je devais avoir 20 ans, je vivais encore à Beyrouth. Je faisais des études de graphisme à l'Académie libanaise des Beaux-Arts. Beyrouth était en pleine reconstruction, avec toute cette problématique de la mémoire très présente pendant cette période. J'ai ressenti un jour l'urgence de raconter un souvenir très précis. Je me suis mise à écrire. Très vite, j'ai éprouvé le besoin de dessiner pour construire l'histoire avec des images et des mots, dans ce rapport du texte à l'image. Je me suis mise à faire de la bande dessinée comme ça, sans préméditation, et ça a donné [Beyrouth] Catharsis, un travail de mémoire, urgent pour moi.

\section{$H \& M$ : En quoi y avait-il pour vous "urgence" à commencer?}

Z. A. : Pour se remettre un peu dans le contexte, le Liban n'a pas fait de travail de mémoire sur la guerre civile, sur les "événements", comme on dit pudiquement là-bas. Dans les livres d'histoire à l'école, le programme s'arrête aux années 1960. Il y a un énorme non-dit. II n'y a pas encore de version officielle, racontable, de cette guerre civile. Pour ce qui est de Beyrouth, comme c'était une guerre civile, les gens se battaient dans les quartiers où ils habitent aujourd'hui. La ville est donc pleine de cette mémoire et pour autant, pas une seule plaque n'indique qu'ici il y a eu tant de morts. II n'y a pas eu du tout de travail de mémoire par rapport à la ville et à ces questions de territoire. Avec la reconstruction du centre-ville, on s'est plutôt appliqué officiellement à donner l'impression qu'il ne s'était jamais rien passé dans ces lieux-là. Comme si on avait posé un vernis sur ces pierres chargées d'histoire. De la même façon, à un niveau plus intime, on parlait peu de cette période dans les familles. Je ne sais plus qui a employé cette formule assez juste : "La guerre du Liban s'est arrêtée comme un match de foot où l'arbitre siffle la fin. Chacun rentre chez soi et c'est plié." C'est assez bien vu. En tout cas, je l'ai vécu comme ça. Ça, c'est le contexte. Le Beyrouth de mon enfance était en train de disparaître, tout comme les traces de ce que nous avions vécu. II me semblait donc important de dire voilà, ça s'est passé.

\section{H\&M : Pourquoi avez-vous choisi le dessin pour raconter ces histoires?}

Z. A. : Je dessinais un peu mais je n'avais aucune confiance dans mon dessin. Or, voilà que je me 
tard, surtout avec Le Jeu des hirondelles, que ce noir et blanc raconte une histoire par lui-même, avec ce que ça induit de vide et de plein dans l'image. II y a, par exemple, une scène où je ne dessine quasiment rien. II y a juste un fond noir avec des dialogues et le noir permet au lecteur d'imaginer la scène, parce que c'est quelque chose d'assez insupportable, d'indicible ou d'“indessinable". De la même façon, le blanc m'a permis aussi d'exprimer le vide de cette ligne de démarcation entre les deux moitiés de la ville. Évidemment, c'était violent, c'était détruit. On peut imaginer des scènes de guerre atroces, mais il n'était pas question de les dessiner. En revanche, le blanc venait dire : "Remplissez le vide vous-mêmes !" Et puis, il y a tout ce jeu d'ornementation, que j'adore, qui vient peut-être de ce que j'ai retenu de la calligraphie arabe. Quand j'étais enfant, je regardais beaucoup la tapisserie, à la maison chez mes parents, et aussi les tapis.

Zeina Abirached, Paris n'est pas une île déserte, 2013. (c) D.R.

suis retrouvée devant cette urgence de raconter. Il fallait que je trouve une façon de raconter l'histoire par les dessins, en dessinant de la manière la plus simple possible. J'ai donc épuré au maximum, en me débarrassant de tout ce que j'étais incapable de faire à l'époque, c'est-à-dire la perspective, en premier lieu. Donc, j'ai opté pour un dessin assez minimaliste. Très vite, je me suis rendu compte que je n'avais pas non plus besoin de la couleur. Non pas parce que je ne savais pas dessiner en couleur, mais parce que ce n'était pas indispensable à la narration. Et puis, j'ai rusé. C'est un enfant qui regarde la scène, donc tous les personnages sont coupés à la taille. Cela me permettait d'exprimer une idée forte dans le cadrage. Rien n'est inutile dans le dessin, ou plutôt rien qui ne soit pas indispensable à l'histoire. Ensuite, mais je m'en suis rendu compte plus
J'étais assez fascinée par tous ces motifs, toutes ces répétitions.

Concernant les influences, je suis passée de la bande dessinée franco-belge - Tintin, Astérix, Lucky Luke, tous ces albums que je connais par cœur et qui ont vraiment compté pour moi - à des auteurs que j'ai découverts un peu sur le tard, toujours dans cette même librairie. Des gens comme Tardi, Hugo Pratt et surtout David B. C'était un vrai choc à l'époque. Voilà quelqu'un qui racontait une histoire autobiographique avec L'Ascension du Haut Mal, en noir et blanc. Hyperpuissant, évidemment, mais ça détonnait vraiment dans ce que je connaissais de la bande dessinée. Pour la première fois, je me suis sentie personnellement proche, non pas de ce qu'il racontait, mais de cet objet dessiné en noir et blanc. C'était un beau point de départ. Je n'ai pas cité José Muñoz. Mais il y a eu Muñoz et Sampayo, évidemment. Je crois me souvenir d'avoir dégoté Billie Holiday dans une braderie. 


\section{COLLECTIONS}

H\&M : Le Musée national de l'histoire de l'immigration a acheté cinq premières planches de quelque chose qui s'appelait à l'origine Paris n'est pas une île déserte, et qui vont finalement être incluses à votre nouvel opus qui sort en 2015. Pouvez-vous nous parler de ce projet?

Z. A. : Au départ, Paris n'est pas une île déserte était une commande du Centre des archives départementales de la région $\mathrm{Paca}$ qui a l'habitude, tous les deux ans, d'inviter un auteur de bande dessinée à réfléchir sur un thème et, du coup, à exposer une trentaine de planches sur le thème en question. On m'avait proposé cette année-là de réfléchir non pas à un thème mais à une mise en regard de mon travail avec celui d'un auteur ou d'un dessinateur libanais. La scène BD au Liban reste encore à défricher. Elle survit, par des publications de qualité d'ailleurs, mais ses auteurs ne sont pas publiés sur place. J'avais invité Michèle Sandjofski, qui était ma professeure de bande dessinée à la fac, à réfléchir sur une exposition. Très vite, nous nous sommes rendu compte que nous avions toutes les deux envie de parler de nos origines. Nous nous sommes donné chacune trente planches. Je ne savais pas du tout ce que je voulais faire. J'avais envie de parler de mon arrièregrand-père qui était drogman (interprète) sous le mandat français au Liban, donc le premier vrai bilingue de la famille qui a navigué toute sa vie entre l'arabe et le français, avec une grande idée du bilinguisme et de la langue française.

J'ai voulu ensuite évoquer ce que cela fait d'avoir le français parmi ses langues maternelles et de vivre pour la première fois en France à 24 ans. Autrement dit, d'avoir déjà tout le bagage que charrie la langue, mais pas encore les codes de la vie dans cette société-là. Ces planches ont été exposées à Aix-en-Provence.

Zeina Abirached, Le Piano oriental.

(c) Casterman, 2015. Planche originale détenue par le Musée national de l'histoire de l'immigration, 2013.45.5.

Ce sont donc des histoires courtes, où j'explore mes rapports avec mes deux langues maternelles. C'est un projet qui était resté un peu dans un tiroir. J'avais commencé l'écriture d'une fiction inspirée de la vie de mon arrière-grand-père maternel, que je n'ai pas connu et qui n’a rien à voir avec l'arrière-grand-père drogman. Dans les années 1950 au Liban, il a inventé un piano oriental. Un piano qui permet de jouer le quart de ton de la musique orientale. C'est un piano droit qui ressemble à tous les autres pianos, mais il y a la pédale de la sourdine, au milieu, qui en fait n'est pas la pédale de la sourdine mais décale toutes 
les cordes à l'intérieur du piano pour arriver à jouer ce quart de ton. Ce piano est donc un instrument bilingue. Je m'étais mise à écrire l'histoire de cet inventeur et de cet instrument extraordinaire qui n'existe qu'en un seul exemplaire au Liban. Le prototype d'usine a été fabriqué à la fin des années 1950 à Vienne par Hoffmann. En cours de route, je me suis rendu compte que ce qui m'intéressait, dans ce piano, cétait son côté bilingue, entre deux cultures, deux modes musicaux, deux langues. Ce que j'avais commencé dans Paris n'est pas une île déserte, j'étais en train de l'écrire aussi à travers ce piano.

Le thème du livre, c'est le langage, ces deux langues, ces deux modes de communication, ces deux cultures. L'histoire est racontée par deux biais : le destin de ce piano et l'exploration de mon rapport à mes langues maternelles. Le titre, c'est Le Piano oriental. II pourrait s'appeler Le Piano bilingue. J'aime bien que les titres soient longs et poétiques. Mais peut-être faut-il faire efficace. J'ai un peu peur du mot "oriental". Je le dis d'ailleurs dans le livre.

\section{H\&M : Problématique, le mot "oriental" ? Parce qu'il charrie des préjugés ?}

Z. A. : Il y a d'abord la réaction basique et assez drôle plutôt liée au fait d'être "libanaise". Pendant mes premières années en France, j'avais l'impression d'être un pois-chiche. À chaque fois qu'on faisait ma connaissance, on me parlait du houmous! Étant libanaise, parlant de cette partie du monde et vivant en France - car je ne suis pas une auteure libanaise qui travaille au Liban, je vis et travaille en France -, je me méfie beaucoup de l'orientalisme des Orientaux. Pas de l'orientalisme des Occidentaux qui fantasment sur l'Orient. Je pense à la forme encore plus pernicieuse de l'orientalisme, qui est l'image de l'Orient véhiculée par les Orientaux eux-mêmes. Je fais très attention à ce que mon écriture véhi- cule par rapport à cette image-là. II ne faut pas que ça tombe dans le folklore, dans quelque chose de trop facilement dit. Ça prend du temps d'expliquer les nuances, mais je pense qu'elles sont très importantes. Ce qui est bien dans la bande dessinée, c'est qu'il y a aussi la dimension de l'humour qui fait que l'on peut dire des choses très graves ou très importantes avec une espèce de pirouette, qui fait que ça capte d'une façon, j'espère, efficace.

\section{H\&M : Certains auteurs refusent de vendre leurs planches. Qu'est-ce qui vous a conduit à accepter cette démarche auprès du Musée national de l'histoire de l'immigration ?}

Z. A. : Très souvent, je retravaille mes planches. Le musée a donc acheté une étape du processus. Le livre n'existait pas encore et c'est ça qui m'a fait beaucoup hésiter. Mais c'était important. Parce que ce travail en particulier m'est important. Il scelle un peu mon rapport à la France, ma naturalisation. Ce sont des choses évidemment très fortes pour moi. II me semblait, au fond, que les planches en question trouvaient ici leur bonne place. Si ça peut contribuer à dire quelque chose à cet endroit-là, alors ça m'intéresse de le faire.

Ma famille vit au Liban. Je n'ai aucune obligation à rester en France. Il y a cette planche, dans Paris n'est pas une île déserte, où l'on voit sur une carte du monde la France et le Liban qui jouent au ping-pong. Je suis constamment entre les deux. J'en suis contente. Je n'ai pas l'air désolé dans le dessin. J'y tiens. J'ai choisi d'être là, je suis heureuse d'être là. En même temps, il n'est pas question de choisir entre les deux. II s'agit vraiment de tisser une histoire, une identité entre ces deux pays, ces deux cultures. II serait peutêtre temps que je mette un mot là-dessus. Mais je ne suis pas sûre que le mot soit "émigration" ou "exil". I 